\title{
ULTRAREGULAR INDUCTIVE LIMITS
}

\section{JAN KUCERA}

\author{
Department of Mathematics \\ Washington State University \\ Pullman, Washington 99164-2930 \\ (Received November 10, 1987 and in revised form February 18, 1988)
}

\begin{abstract}
An inductive limit $E=\operatorname{ind} \lim E_{n}$ is ultraregular if it is regular and each set $B \subset E_{n}$, which is bounded in $E$, is also bounded in $E_{n}$. A necessary and sufficient condition for ultraregularity of $E$ is given provided each $E_{n}$ is an $L F$-space which is closed in $E_{n+1}$.
\end{abstract}

KEY WORDS AND PHRASES. Regular and ultraregular inductive limits, bounded set, fast completeness.

MATHEMATICS SUBJECT CLASSIFICATION. Primary 46 A 12, Secondary 46 A06.

Let $F$ be a locally convex space and $B \subset F$ an absolutely convex subset. We denote by $F_{B}$ the linear hull of $B$ and provide it with the topology generated by the Minkowski functional of $B$. If the toplogical space $F_{B}$ is Banach then $B$ is called a Banach disk. In [1] de Wilde calls the space $F$ fast complete if every set $B$, bounded in $F$, is contained in a bounded Banach disk. Every sequentially complete space is fast complete.

A strict inductive limit of a sequence $F_{1} \subset F_{2} \subset \cdots$ of Fréchet spaces is called an $L F$-space.

If $S \subset X \cap Y$, where $X$ and $Y$ are locally convex spaces, then $c \ell_{X} S$ and $c \ell_{Y} S$ are the respective closures of $S$ in $X$ and $Y$. Throughout the paper $E_{1} \subset E_{2} \subset \cdots$ is a sequence of Hausdorff locally convex spaces with all inclusions id: $E_{n} \rightarrow E_{n+1}$ continuous. We denote indlim $E_{n}$ by $E$.

In [2] Floret calls an inductive limit $E$ regular, resp. $\alpha$-regular, if every set bounded in $E$ is bounded, resp. contained, in some $E_{n}$. An $\alpha$-regular inductive limit is ultraregular, resp. weakly ultraregular, if each set $B \subset E_{n}$, which is bounded in $E$, is also bounded, resp. weakly bounded, in $E_{n}$.

In $[3, \S 4$, Prop. 4] Dieudonné and Schwartz proved that a strict inductive limit is ultraregular if each space $E_{n}$ is closed in $E_{n+1}$. In the casc the inductive limit is not strict some restrictions on topologies of the spaces $E_{n}$ have to be imposed.

We introduce two properties:

(P) Every closed absolutely convex neighborhond in $E_{n}$ is closed in $E_{n+1}$. 
(Q) If $U$ is a closed absolutely convex neighborhood in $E_{n}$ and $V$ is the closure of $U$ in $E_{n+1}$, then $U=V \cap E_{n}$.

Evidently $P \Rightarrow Q$ and if each space $E_{n}$ is closed in $E_{n+1}$ then $P$ is equivalent to $Q$. If the inductive limit $E$ is strict then $Q$ holds.

Lemma 1. (Q) holds iff each real $f \in E_{n}^{\prime}$ has a real continuous linear extension to $E_{n+1}$.

Proof. Assume $(Q)$ and take a real $f \in E_{n}^{\prime}, f \neq 0$. The set $U=f^{-1}[-1,1]$ is a closed absolutely convex neighborhood in $E_{n}$. Let $V$ be the corresponding set $V \subset E_{n+1}$ from $(Q)$. If $E_{n} \subset V$ we would have $U=V \cap E_{n}=E_{n}$ and $f=0$, which contradicts our assumption $f \neq 0$. Hence we can choose $x_{0} \in E_{n} \mid V$. Since $V$ is absolutely convex and closed in $E_{n+1}$, there exists a real $g \in E_{n+1}^{\prime}$ such that $V \subset g^{-1}(-\infty, 1]$ and $g\left(x_{0}\right)>1$. Further $f^{-1}(0) \subset g^{-1}(0)$ which implies $f\left(x_{0}\right) \neq 0$. Without a loss of generality we may assume $f\left(x_{0}\right)=1$. Then $f\left(x-f(x) x_{0}\right)=0$ for $x \in E_{n}$ and $\left(x-f(x) x_{0}\right) \in f^{-1}(0) \subset g^{-1}(0)$. Hence, $g\left(x-f(x) x_{0}\right)=g(x)-f(x) g\left(x_{0}\right)=0$ and the functional $\left(g\left(x_{0}\right)\right)^{-1} g \in E_{n+1}^{\prime}$ is the desired extension.

Assume that each real $f \in E_{n}^{\prime}$ has a real extension $g \in E_{n+1}^{\prime}$. Take a closed absolutely convex neighborhood $U \subset E_{n}$. By the Hahn-Banach theorem there exists $F \subset E_{n}^{\prime}$ such that each $f \in F$ is real and $U=\cap\left\{f^{-1}(-\infty, 1) ; f \in F\right\}$. Let $G$ be the set of all real extensions of all $f \in F$ to $E_{n+1}$. The set $V=\cap\left\{g^{-1}(-\infty, 1] ; g \in G\right\}$ is closed and absolutely convex in $E_{n+1}$. Evidently $U \subset V \cap E_{n}$. Assume $U \neq V \cap E_{n}$. Then there is $y \in\left(V \cap E_{n}\right) \backslash U \subset E_{n} \backslash U$ and $f \in F$ such that $y \notin f^{-1}(-\infty, 1]=E_{n} \cap g^{-1}(-\infty, 1]$, where $g \in G$ is an extension of $f$. But then $y \notin E_{n} \cap V \subset E_{n} \cap g^{-1}(-\infty, 1]$, which is a contradiction. Hence $U=V \cap E_{n}$ and $(Q)$ holds.

Lemma 2. $(P) \Rightarrow E \alpha$-regular.

Proof. Assume that $E$ is not $\alpha$-regular. Then there is a set $B$ bounded in $E$ which is not contained in any $E_{n}$. By taking a subsequence of $E_{1}, E_{2}, \cdots$, we may assume that for any $n \in N$ there exists $b_{n} \in\left(B \cap E_{n}\right) \backslash E_{n-1}, E_{0}=\{0\}$.

Since $b_{1} \neq 0$, there is a closed absolutely convex neighborhood $U_{1}$ in $E_{1}$ such that $b_{1} \notin U_{1}$. Also $b_{2} \notin E_{1}$. Hence $\frac{1}{2} b_{2} \notin U_{1}$. By $(P), U_{1}$ is closed in $E_{2}$ and there exists an absolutely convex neighborhood $V_{1}$ in $E_{2}$ such that $\left(b_{1}+V_{1}+V_{1}\right) \cap U_{1}=\emptyset$ and $\left(\frac{1}{2} b_{2}+V_{1}+V_{1}\right) \cap U_{1}=\emptyset$. Then $U_{2}=c \ell_{E_{2}}\left(U_{1}+V_{1}\right)$ is a closed absolutely convex neighborhood in $E_{2}$ such that $b_{1}, \frac{1}{2} b_{2} \notin U_{2}$. Again $U_{2}$ is closed in $E_{3}$ and $\frac{1}{3} b_{3} \notin U_{2}$. Hence there is an absolutely convex neighborhood $V_{2}$ in $E_{3}$ such that $\left(\frac{1}{k} b_{k}+V_{2}+V_{2}\right) \cap U_{2}=\emptyset$ for $k=1,2,3$. The set $U_{3}=c \ell_{E_{3}}\left(U_{2}+V_{2}\right)$ is a closed absolutely convex neighborhood in $E_{3}$ for which $\frac{1}{k} b_{k} \notin U_{3}, k=1,2,3,4$.

Once all such $U_{n}, n \in N$, are constructed, $U\left\{U_{n} ; n \in N\right\}$ is a neighborhood in $E$ which does not absorb $B$, a contradiction. 
Lemma 9. $(P) \Rightarrow E$ weakly ultraregular.

Proof. Assume $(P)$ and $E$ not weakly ultraregular. By I,emma $2, E$ is $\alpha$-regular. Hence, there exists a set $B$ bounded in $E$ and $n \in N$ such that $B \subset E_{n}$ but $B$ is not weakly bounded in $E_{n}$. Without a loss of generality we may assume $n=1$.

Take a real $f_{1} \in E_{1}^{\prime}$ which is not bounded on $B$ and choose a sequence $b_{n} \in B, n \in N$, such that $f_{1}\left(b_{n}\right)>n$. Since $(P)$ implies $(Q)$ there is a real extension $f_{2} \in E_{2}^{\prime}$ of $f_{1}$ and a real extension $f_{3} \in E_{3}^{\prime}$ of $f_{2}$, etc. Each set $U_{n}=f_{n}^{-1}(-\infty, 1], n \in N$, is a closed absolutely convex neighborhood in $E_{n}$ and $U_{1} \subset U_{2} \subset \ldots$. Hence $U=U\left\{U_{n} ; n \in N\right\}$ is a 0-neighborhood in $E$. For any $n \in N$ we have $b_{n} \notin n \cup$, i.e. $U$ does not absorb $B$ which is a contradiction.

Theorem 1. Let $(P)$ hold and each $E_{n}$ be fast complete. Then $E$ is ultraregular.

Proof. By Lemma 2, $E$ is $\alpha$-regular. Let $B \subset E$ be bounded. Then $B \subset E_{n}$ for some $n \in N$. By Lemma $3, B$ is weakly bounded in $E_{n}$. Since $E_{n}$ is fast complete, $B$ is also bounded with respect to the topology of $E_{n}$, see [4].

Lemma 1. Let each $E_{n}$ be an inductive limit of metrizable spaces and $E$ ultraregular. Then $(Q)$ holds.

Proof. Take a real $f \in E_{1}^{\prime}, f \neq 0$. It suffices to show that $f$ has a continuous linear extension to $E_{2}$. Put $F=\left(E_{1}\right.$, top $\left.E_{2}\right)$. Since the inclusion id: $E_{1} \rightarrow F$ is continuous, each set bounded in $E_{1}$ is bounded in $F$. On the other hand if $B$ is bounded in $F$ it is bounded in $E$ and $B \subset E_{1}$. Then $B$ is bounded in $E_{1}$ by the ultraregularity of $E$. Hence the spaces $E_{1}$ and $F$ have the same families of bounded sets.

The set $A=f^{-1}(-1,1)$ absorbs all sets bounded in $E_{1}$, hence it absorbs all sets bounded in $F$. The space $F$, as an inductive limit of metrizable spaces, is bornological. This implies $A$ is a 0-neighborhood in $F$. If $a<b, x_{0}\left\ulcorner f^{-1}(a, b)\right.$, and $d=\min \left(f\left(x_{0}\right)-a, b-f\left(x_{0}\right)\right)$, then $d>0$ and $x_{0}+d \boldsymbol{A} \subset f^{-1}(a, b)$. Thus $f^{-1}(a, b)$ is open in $F$ and $f^{-1}(-\infty, 1]=F \backslash \cup\left\{f^{-1}(1, n) ; n \in N^{-}\right\}$is closed in $F$. The set $\left.M=c \ell_{E_{2}} f^{1}(-\infty), 1\right]$ is closed absolutely convex in $E_{2}$ and $f^{-1}(\infty, 1]=$ $M \cap F=M \cap E_{1}$.

Take $x_{1} \in E_{1}$ for which $f\left(x_{1}\right)>1$. Then $x_{1} \notin M$ and there exists a real $g \in E_{2}^{\prime}$ such that $M \subset g^{-1}(-\infty, 1]$ and $g\left(x_{1}\right)>1$. If $x+f^{-1}(0)$ then $f(k x)=0$ for each integer $k$ which implies $k x \in M$ and $x \in g^{-1}(0)$. Hence $f^{-1}(0) \subset g^{-1}(0)$ ind there exists $c>0$ such that $c g(x)=f(x)$ for $x \in E_{1}$. The functional $c g$ is the sought linear continuous extension of $f$ to $E_{2}$.

Theorem 2. Assume

1. Each $E_{n}$ is closed in $E_{n+1}$.

2. Each $E_{n}$ is an inductive limit of metrizible spaces.

3. $E$ is ultraregular.

Then $(P)$ holds. 
Proof. By Lemma 4, assumptions 2 and 3 imply $(Q)$ which combined with the assumption in 1 implies $(P)$.

Theorem 9. Assume

1. Each $E_{n}$ is closed in $E_{n+1}$.

2. Each $E_{n}$ is $L F$-space.

Then $E$ is ultraregular iff $(P)$ holds.

Proof. The if part follows from Theorem 2. For the only if part we observe that each $L F$-space $E_{n}$ satisfies the assumptions of the Dieudonné-Schwartz Theorem in [3]. Hence $E_{n}$ is ultraregular and therefore also regular. Since regular inductive limit, not necessarily strict, of Fréchet spaces is fast complete, [5], each space $E_{n}$ is fast complete. Then, by Theorem 1 , $(P)$ implies the ultraregularity of $E$.

\section{References}

[1] Marc de Wilde, Doctoral Dissertation, Mém. Soc. R. Sc., Liege, 18, 2, 1969.

[2] Klaus Floret, On Bounded Sets in Inductive Limits of Normed Spaces, Proc. AMS, 75, 2, 1979, 221-225.

[3] Jean Dieudonné, Laurent Schwartz, La dualité dans les espaces (F) et (LF), Ann. Inst. Fourier (Grenoble) 1, 1949, 61-101.

[4] Carlos Bosch, Jan Kucera, A necessary and sufficient condition for w*-bounded sets to be strongly bounded, Proc. AMS, 101, 3, 1987, 453-454.

[5] Jan Kucera, Carlos Bosch, Bounded sets in fast complete inductive limits, Int. J. Math. \& Math. Sci. 7, 3, 1984, 615-617. 


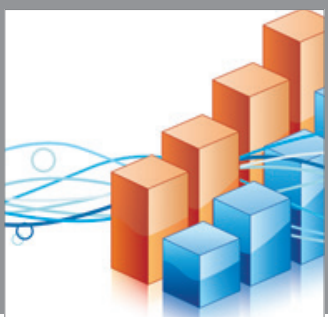

Advances in

Operations Research

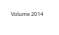

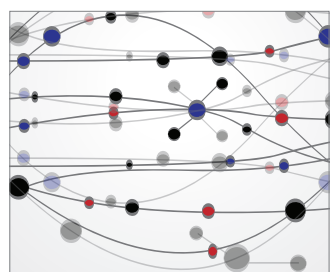

\section{The Scientific} World Journal
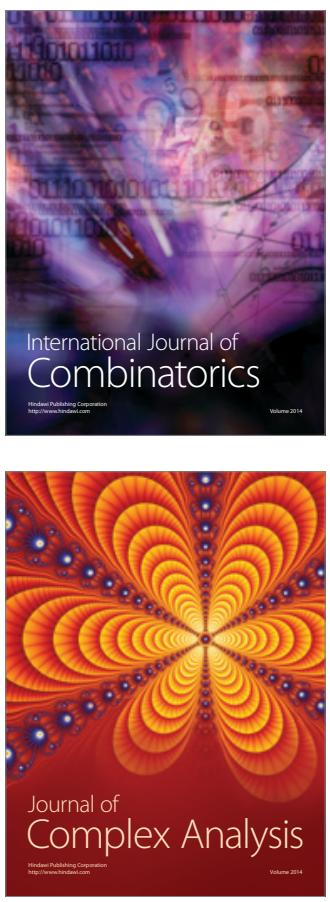

International Journal of

Mathematics and

Mathematical

Sciences
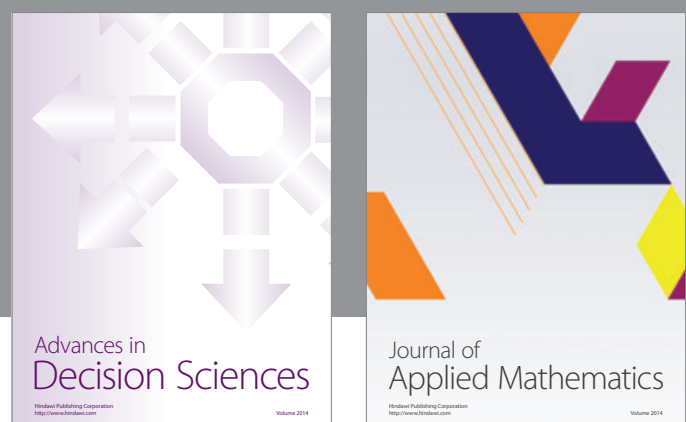

Journal of

Applied Mathematics
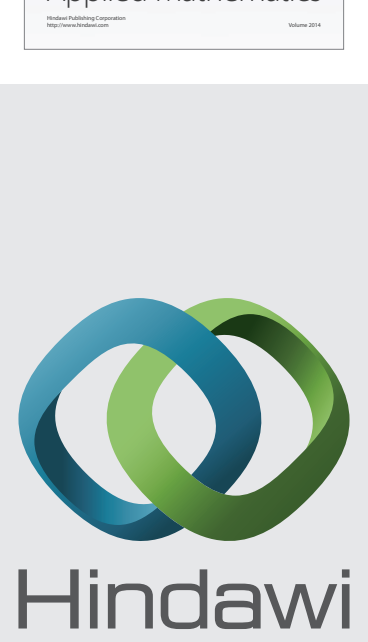

Submit your manuscripts at http://www.hindawi.com
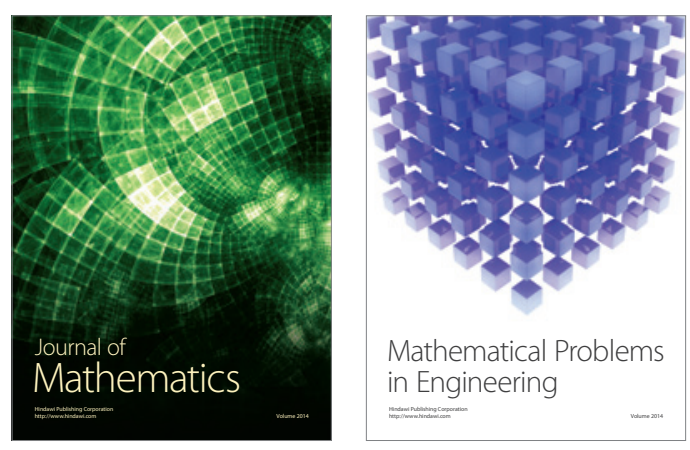

Mathematical Problems in Engineering
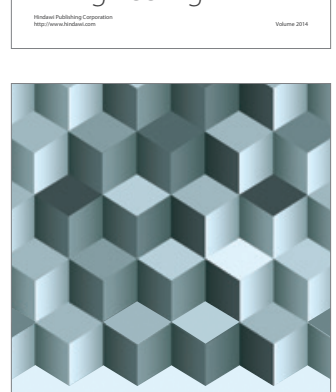

Journal of

Function Spaces
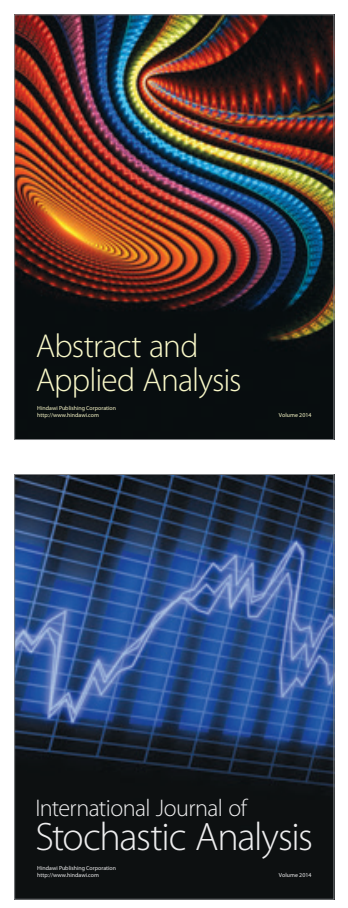

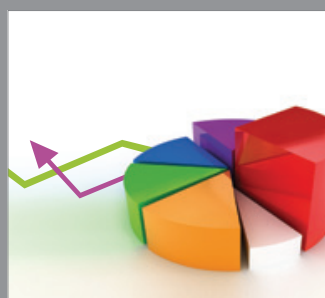

ournal of

Probability and Statistics

Promensencen
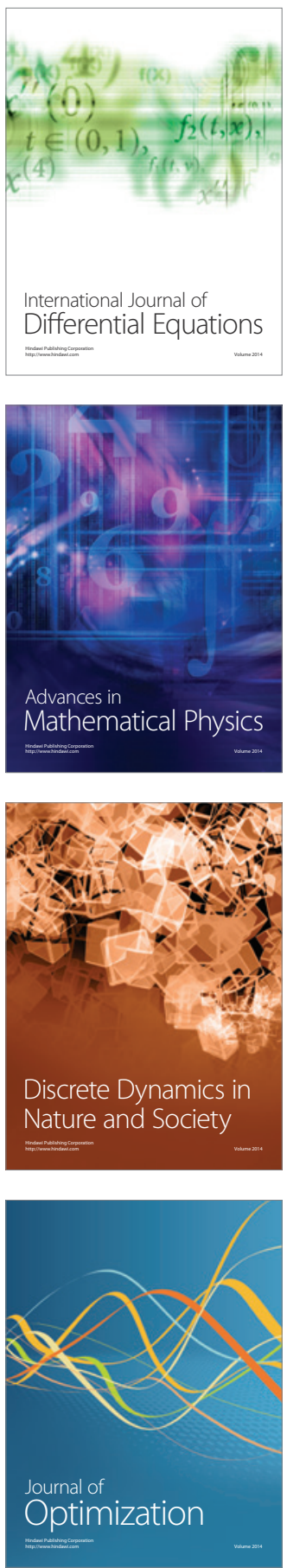